\title{
Research on Strain Rate-Dependent Tsai-Hill Strength Criterion for Ethylene Propylene Diene Monomer Film
}

\author{
Xu Jinsheng, Yu Jiaquan, Chen Xiong* , Niu Ranming and Jia Deng \\ School of Mechanical Engineering, Nanjing University of Science and Technology, Nanjing, 210094, China
}

\begin{abstract}
EPDM film is an innovative insulation material used in the Solid Rocket Motor. İt is a kind of unidirectional single layer fiber-reinforced composite plate. To analyze its mechanical behavior uniaxial tests with different loading directions and rates were performed. The test results indicated that the EPDM film is anisotropic and its mechanical properties are obviously rate-dependent. To create a rate-dependent anisotropic strength criterion, two methods were adopted to extend the Tsai-Hill strength criterion; the first was to substitute all the strength components in the Tsai-Hill criterion with the fitted rate-dependent ones, the second was to introduce a special factor named the rate-dependency factor $R(\dot{\varepsilon})$ into the criterion. The second method is simpler in form and more suitable for an engineering application. The two rate-dependent criteria were examined by comparing the predicted results with the experimental ones, and the results showed good agreement which verified that the strength criteria were able to reveal the anisotropic rate-dependent properties of the EPDM film.
\end{abstract}

Keywords: Breaking strength, EPDM, strength criterion, uniaxial elongation.

\section{INTRODUCTION}

Insulation is an important component in the Solid Rocket Motor (SRM). It controls the variation of the burning area of the grain and determines the internal ballistics property of the SRM. Extraordinary adiabatic and anti- thermal ablation properties are the basic requirements for the insulation to coat the grain, but the insulation should also fulfill certain mechanical requirements. With these mechanical properties the insulation will be able to ensure its structural integrity during manufacturing, storing, transporting and serving. Thus, various types of loading, including impact, vibration, and creep will challenge the mechanical limits of the insulation material.

Generally speaking the insulation is a kind of polymer composites consisting of matrixed materials, functional brasques, polymer adhesives, and other additions. Commonly used insulation materials fall into three categories: organic silicon rubber, polyurethane elastomer, and Ethylene Propylene Diene Monomer (EPDM). EPDM rubber is widely used because of its relatively low density, long storage property and tougher ablation property, EPDM film is a kind of unidirectional single layer fiber-reinforced composite plate; with the stacking techniques for resin impregnation layers of EPDM films can form an advanced laminated insulation material [1]. The general theory says that the lamination strength can be predicted once the basic strength characteristics of a single unidirectional layer have been determined [2]. To determine the anisotropic strength of the composites, Tsai $\mathrm{W}$. used a strength criterion which was deduced from the one given by Hill [3]. However, none

*Address correspondence to this author at the School of Mechanical Engineering, Nanjing University of Science and Technology, Nanjing, 210094, China; Tel: 13913884552; E-mail: xujinsheng@njust.edu.cn of these criteria consider the rate-dependency of the material; yet the viscoelastic property is one of the main properties of EPDM film. Recently, Ming and Weinong [4] built Ogden and Roxburgh's models after testing under quasi-static and dynamic loading rates. Besides the rate effect, their research also investigated the influence of the Mullins effect. Song [5] investigated the dynamic compressive behavior of EPDM rubber with a Hopkinson pressure bar and built a ratedependent model by modifying an energy based onedimensional dynamic constitutive equation. Currently EPDM is of increasing interest to researchers, and it has brought more and more advanced experimental methods to the field, e.g. Thermogravimetry Analysis (TGA), artificial aging [6], and Transmission electron microscopy (TEM) [7].

In this study, uniaxial elongation tests were made with the specimens cut along 7 different directions to study the anisotropic properties of EPDM film. On each occasion four loading rates were chosen to analyze the effects of the strain rate on the strength. The purpose of this study was to determine the rate-dependent anisotropic strength criterion of EPDM film, thus providing basic fundamental research for investigating laminated EPDM in the future. Once the necessary experimental results were finished we used two methods to improve the Tsai Hill strength criteria. One method substituted all the strengths in Tsai Hill equation with the rate-dependent ones. The other one introduced a rate-dependent factor into the equation. The strength criteria were created and verified.

\section{EXPERIMENT AND RESULTS}

\subsection{Material and Methodology}

The tested EPDM film is $0.5 \pm 0.02 \mathrm{~mm}$ thick and is a new type of propellant coating material. Its anisotropic 
mechanical behavior results from the single orientation fibers which lay uniformly across its polymer matrix. To fully understand the mechanical properties of the material the orientation of the fibers is assumed to be $0^{\circ}$ direction. The material is cut into $80 \mathrm{~mm} \times 10 \mathrm{~mm}$ strips, each with a test length of $40 \mathrm{~mm}$ along 7 directions, which are $0^{\circ}, 15^{\circ}, 30^{\circ}$, $45^{\circ}, 60^{\circ}, 75^{\circ}, 90^{\circ}$, respectively, as shown in Fig. (1).
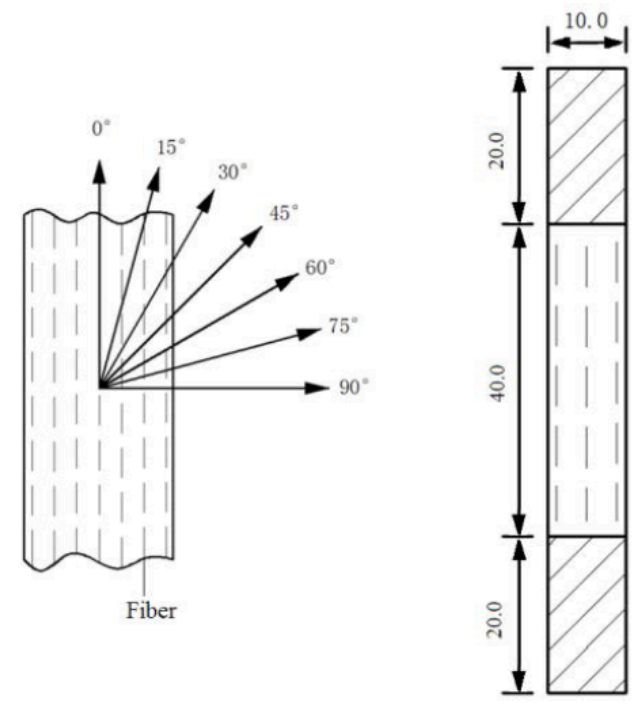

Fig. (1). Fiber distribution and specimen geometry.

\subsection{Experiment}

As mentioned above specimens were cut into 7 specimens with different offset angles. Uniaxial tests with these specimens were conducted under different rates to analyze the influence of the loading direction and loading rate on the mechanical property of the EPDM film. With a relative humidity of $40 \%$ and the temperature of $25^{\circ} \mathrm{C}$, three sustainable tests were conducted with each specimen under loading rates of $5,20,100$, and $500 \mathrm{~mm} / \mathrm{min}$.

\subsection{Results}

Fig. (2) shows the three uniaxial tests of the $90^{\circ}$ specimen under the loading rate of $500 \mathrm{~mm} / \mathrm{min}$ and also shows the averaged results. This illustrates an example of the data processing procedure for all the other specimens under the four loading rates.

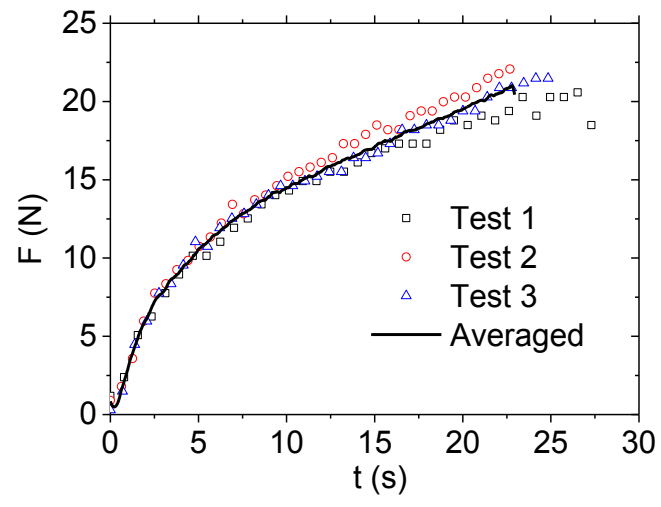

Fig. (2). $90^{\circ}$ Specimen under a loading rate of $500 \mathrm{~mm} / \mathrm{min}$.
The Stress-Strain curves of the $5 \mathrm{~mm} / \mathrm{min}, 20 \mathrm{~mm} / \mathrm{min}$, $100 \mathrm{~mm} / \mathrm{min}$, and $500 \mathrm{~mm} / \mathrm{min}$ uniaxial tests with $7 \mathrm{different}$ loading directions were illustrated in Fig. (3a-d), respectively.

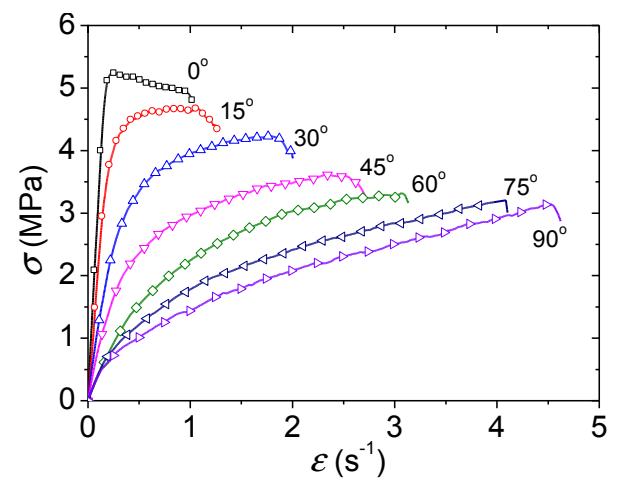

(a) $5 \mathrm{~mm} / \mathrm{min}$

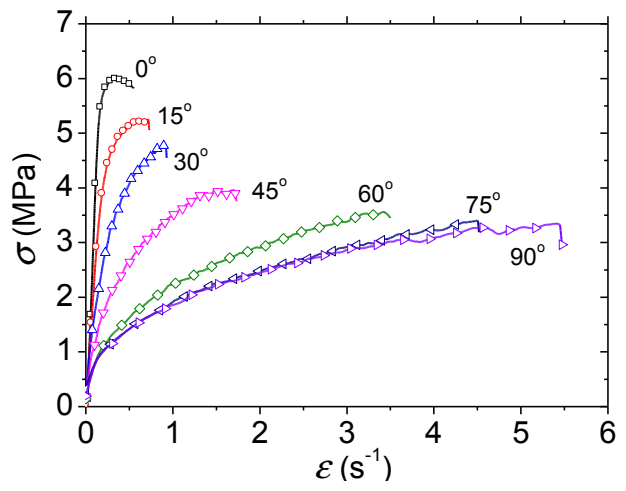

(b) $20 \mathrm{~mm} / \mathrm{min}$

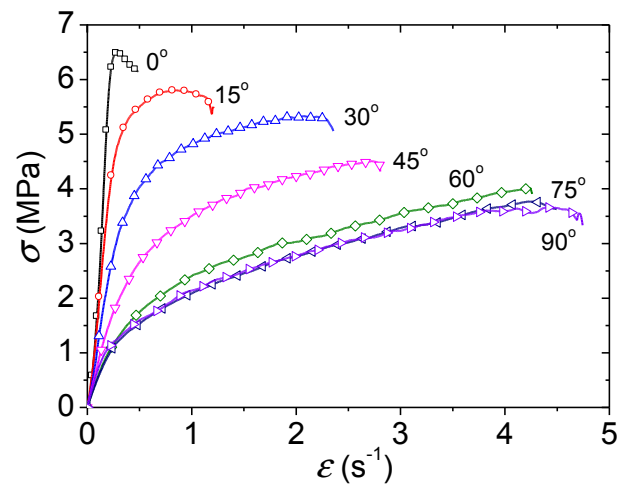

(c) $100 \mathrm{~mm} / \mathrm{min}$

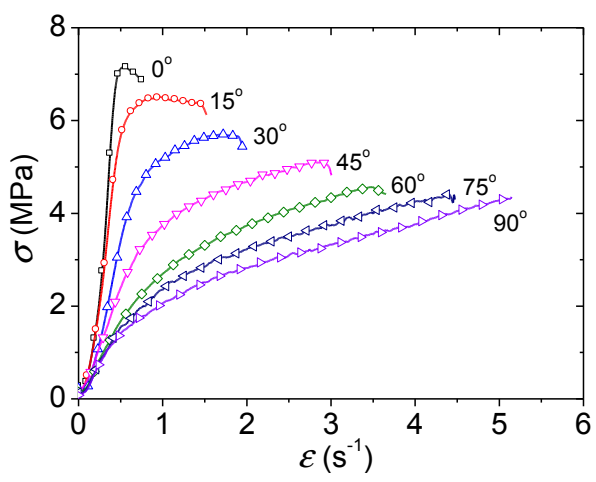

(d) $500 \mathrm{~mm} / \mathrm{min}$

Fig. (3). Test results along different loading directions. 
This shows that the ductility increases with the increase of the loading angle. In the case of loading along the same direction, the strength of the material will grow when the loading rate increases. When loaded with the same rate and the loading angle ranges between $0^{\circ}$ and $60^{\circ}$, the strength of the material goes up with the increase of the loading angle; however, when the loading angle is between $60^{\circ}$ and $90^{\circ}$, the strength of the material is constant.

A possible cause for this phenomenon is that the modulus and fiber strength are much higher than the ones in the matrix, and fiber is the main factor in the mechanical property when the loading angle is small. However, when the loading angle increased, weakening the function of the fiber, there was no obvious change in the mechanical property of EPDM. Also the addition of the fiber would restrict the stretch of the molecular chain inside the matrix, which makes the overall ductility fall, especially in the case of loading at a small angle.

\section{STRENGTH CRITERION}

The EPDM film is a kind of anisotropic and ratedependent material according to its micro structure, thus an anisotropic rate-dependent strength criterion should be built to characterize the fracture property of this kind of EPDM film.

\subsection{Tsai-Hill Strength Criterion}

Based on the isotropy Mises yield criterion, Tsai-Hill expanded the criterion into the field of orthogonal anisotropic materials by importing several anisotropic parameters. For the EPDM film a single direction film, i.e. $\sigma_{3}=\tau_{23}=\tau_{31}=0$, (in which 1 represents the orientation of the fibers, and 3 represents the normal direction of the film), the yield criterion can be expressed as:

$$
(G+H) \sigma_{1}^{2}+(F+H) \sigma_{2}^{2}-2 H \sigma_{1} \sigma_{2}+2 N \tau_{12}^{2}=1
$$

where, $F, G, H, N$ are the anisotropic parameters, and $\sigma_{1} 、 \sigma_{2} 、 \tau_{12}$ are the stress components of the three directions of the material.

The anisotropic parameters are determined by 6 basic strength parameters, which include three principle directions, $\mathrm{X}, \mathrm{Y}$ and $\mathrm{Z}$ and three shear directions, $S_{23}, S_{31}, S_{12}$. With respect to the fiber strength of the EPDM film, directions 2 and 3 are both normal to the orientation of the fibers. According to the symmetry of the material, the strength of the film along both directions should be the same, that is to say, $Y=Z$. Thus, according to the yield criterion conditions of the orthotropic material under simple stress states, we obtain the following set of equation (2).

$$
\begin{gathered}
(G+H)=\frac{1}{X^{2}} \\
(F+H)=\frac{1}{Y^{2}} \\
2 H=\frac{1}{X^{2}} \\
2 N=\frac{1}{S^{2}}
\end{gathered}
$$

Substitute equation (2) into equation (1). Then the expression of the Tsai-Hill strength criterion of the orthotropic single direction film becomes equation (3):
$\frac{\sigma_{1}^{2}}{X^{2}}-\frac{\sigma_{1} \sigma_{2}}{X^{2}}+\frac{\sigma_{2}^{2}}{Y^{2}}+\frac{\tau_{12}^{2}}{S^{2}}=1$

For EPDM film the loading direction cannot be guaranteed to fall entirely along the principal direction of the film, thus an angle $\theta$ is defined as the angle between the loading direction and the orientation of the fibers. The coordinate transformation of the stress components is given by equation (4):

$$
\begin{gathered}
\sigma_{1}=\sigma_{x} \cos ^{2} \theta \\
\sigma_{2}=\sigma_{x} \sin ^{2} \theta \\
\tau_{12}=\sigma_{x} \sin \theta \cos \theta
\end{gathered}
$$

By substituting equation (4) into the Tsai-Hill strength criterion of the orthotropic unidirectional film under a simple single direction loading we get equation (5):

$\frac{\cos ^{4} \theta}{X^{2}}+\left(\frac{1}{S^{2}}-\frac{1}{X^{2}}\right) \sin ^{2} \theta \cos ^{2} \theta+\frac{\sin ^{4} \theta}{Y^{2}}=\frac{1}{\sigma_{x}^{2}}$

\subsection{Rate-Dependent Strength Criterion}

The experiment shows that an appearance of ratedependency occurs when the EPDM is damaged along different directions, hence it's necessary to bring in the ratedependency of the Principal direction strength, $X$ and $Y$, and the shear strength $c$ when building the Tsai-Hill strength criterion for the EPDM film. X, Y and $S_{x y}$ can be expressed as the functions of the strain rate according to equation (6):

$$
\begin{aligned}
\mathrm{X} & =\mathrm{X}(\dot{\varepsilon}) \\
\mathrm{Y} & =\mathrm{Y}(\dot{\varepsilon}) \\
\mathrm{S}_{X Y} & =\mathrm{S}_{X Y}(\dot{\varepsilon})
\end{aligned}
$$

According to equation (5) the rate-dependent form of the Tsai-Hill strength criterion can be expressed as equation (7) when the unidirectional fiber reinforced plate is loaded by a single direction force:

$\frac{\cos ^{4} \theta}{X(\dot{\varepsilon})^{2}}+\left(\frac{1}{S(\dot{\varepsilon})^{2}}-\frac{1}{X(\dot{\varepsilon})^{2}}\right) \sin ^{2} \theta \cos ^{2} \theta+\frac{\sin ^{4} \theta}{Y(\dot{\varepsilon})^{2}}=\frac{1}{\sigma_{x}(\dot{\varepsilon})^{2}}$

In equation (6) the axial strength along the principal directions of the material under any strain rate can be easily obtained by uniaxial tests under a corresponding loading rate. Using this method the variations of X, Y, and $S_{x y}$ are calibrated and illustrated in Fig. (4).

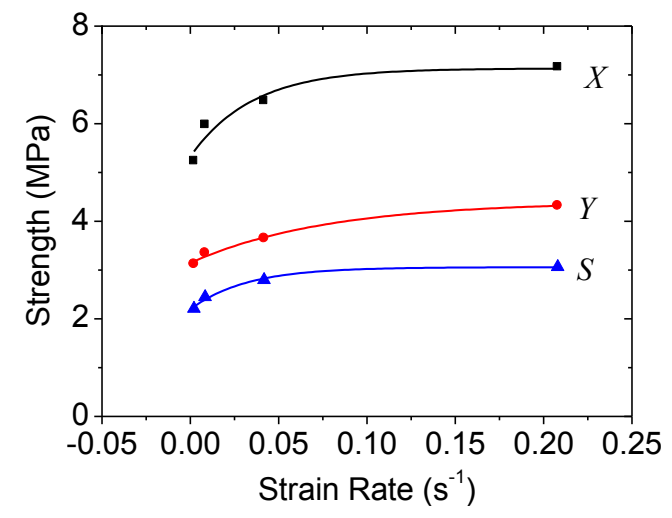

Fig. (4). The variation of axial strength $\mathrm{X}, \mathrm{Y}$, and shear strength $S_{x y}$ with the strain rate.

By averaging the results of the uniaxial tests where the loading direction is deflected by an angle $\theta$ from the fiber 
orientation, the strengths can be obtained by fitting equation (7). Fig. (5) shows how the strengths change with the strain rate.

Fig. (5) illustrates the significant effect that when the strain rate goes up, strengths $\mathrm{X}, \mathrm{Y}$, and $\mathrm{S}$ all increase, but increase more slowly than the strain rate rises. Overall, the axial strengths and the shear strength can be fitted as an exponential function using the strain rate as the variable. The strength laws are given by equation (8):

$$
\begin{aligned}
\mathrm{X} & =7.13\{1-\exp [-28.47 \cdot(\dot{\varepsilon}+0.048)]\} \\
\mathrm{Y} & =4.4\{1-\exp [-12.94 \cdot(\dot{\varepsilon}+0.097)]\} \\
\mathrm{S} & =3.06\{1-\exp [-31.53 \cdot(\dot{\varepsilon}+0.04)]\}
\end{aligned}
$$

The strength of the EPDM at different loading directions can be predicted according to the three strength laws and equation (7). This is shown in Fig. (5), and the estimated results show good agreement with the experimental results, which verifies that the strengths along the different directions were appropriately predicted. It can also be seen that the strength limit becomes smaller as the angle between the loading direction and the fiber orientation goes up. Occasionally the decrease steepens when the angle varies between 0 and 45 , and flattens when the angle varies between 45 and 90 .

When the result is combined with the experimental data, the EPDM substrate shows a robust flexibility and a flimsy strength, but the fiber inside the film shows the opposite property, meaning a robust strength and a poor ductility. For this reason, the strength of the EPDM film can be deeply affected by the fibers when the loading direction is close to their orientation, i.e. $0^{\circ}<\theta<45^{\circ}$. However, when $\theta$ is between $45^{\circ}$ and $90^{\circ}$, the film is likely to be affected by the matrix rather than the fibers. The higher the $\theta$ is, the more dominant role the matrix will play in determining the mechanical property of the EPDM film and the less effective the fiber becomes.

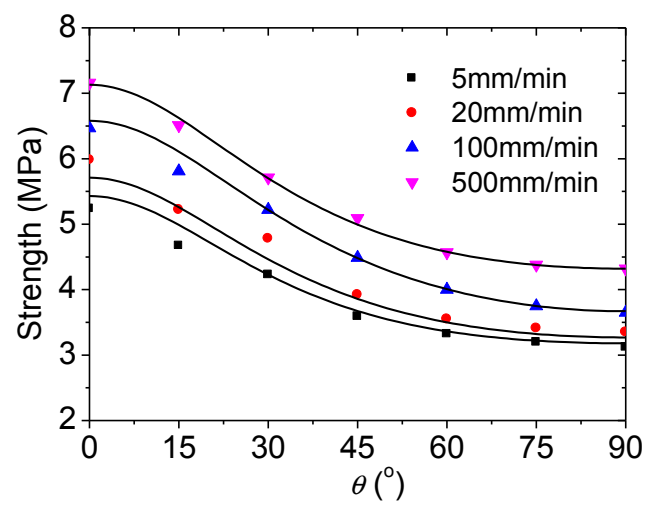

Fig. (5). The predicted strength limits of EPDM film under different loading direction.

According to the experimental results, the EPDM film has a stress law similar to the variation in the strain rate in different loading directions. Thus, a rate-dependent factor $R(\dot{\varepsilon})$ can be introduced into equation (3) to create equation (9):

$\frac{1}{R(\dot{\varepsilon})^{2}} \cdot\left(\frac{\sigma_{1}^{2}}{X^{2}}-\frac{\sigma_{1} \sigma_{2}}{X^{2}}+\frac{\sigma_{2}^{2}}{Y^{2}}+\frac{\tau_{12}^{2}}{S^{2}}\right)=1$
Here, the rate-dependent factor $R(\dot{\varepsilon})$ changes with the strain rate. when the EPDM film is loaded by a uniaxial force, the Tsai-Hill rate-dependent strength law becomes equation (10):

$\frac{\cos ^{4} \theta}{X^{2}}+\left(\frac{1}{S^{2}}-\frac{1}{X^{2}}\right) \sin ^{2} \theta \cos ^{2} \theta+\frac{\sin ^{4} \theta}{Y^{2}}=\frac{R(\dot{\varepsilon})}{\sigma_{x}^{2}}$

The rate-dependent factor $R(\dot{\varepsilon})$ can be obtained by testing with different strain rates at a single loading direction. $R(\dot{\varepsilon})$ was assumed to be 1 when loaded with the reference speed, which was chosen to be $100 \mathrm{~mm} / \mathrm{min}$. Thus the axial strength $\mathrm{X}, \mathrm{Y}$ and the shear strength $\mathrm{S}$ remain constant as the strain rates vary over $6.47 \mathrm{MPa}, 3.65 \mathrm{MPa}$, and $2.80 \mathrm{MPa}$, respectively. The other rate-dependent factor under other loading rates can be obtained using equation (7) to give equation (11) :

$\frac{R\left(\dot{\varepsilon}_{2}\right)}{\sigma_{\chi}^{2}\left(\dot{\varepsilon}_{2}\right)}=\left.\frac{R\left(\dot{\varepsilon}_{1}\right)}{\sigma_{\chi}^{2}\left(\dot{\varepsilon}_{1}\right)}\right|_{100 \mathrm{~mm} / \mathrm{min}}$

Given one single strain rate 7 rate-dependent factors can be obtained using the results of the 7 tests along different loading directions. By fitting the averaged results, the ratedependent factor shows a positive correlation with the strain rate, but rises more slowly with the increase of the strain rate, as shown in Fig. (6).

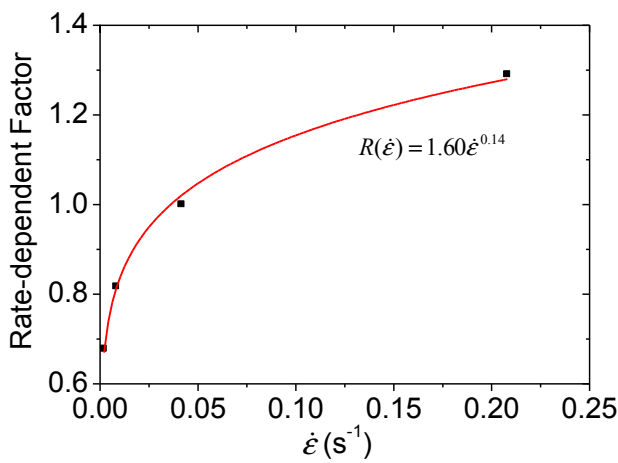

Fig. (6). Rate-Dependency factors.

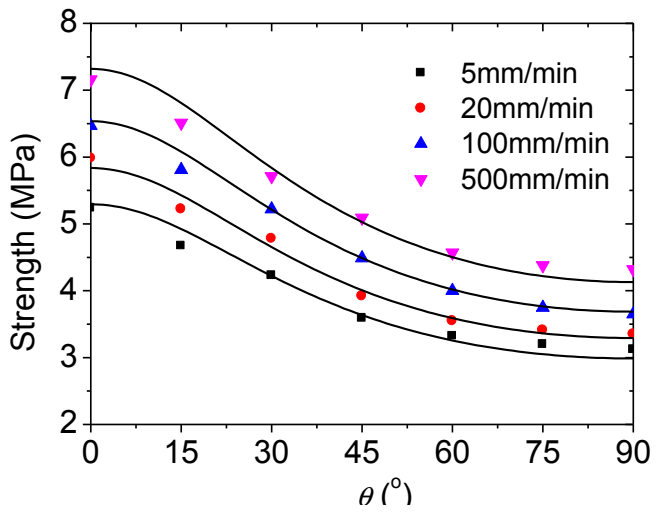

Fig. (7). Strength with different loading directions and strain rates.

The strength of EPDM film loaded with different directions and strain rates can be predicted by equation (7), as shown in Fig. (7). By introducing the rate-dependent factor, the orthotropy and the rate-dependency of the film can be accurately described, which is a more practical and obviously simpler way than establishing $\mathrm{X}, \mathrm{Y}$ and $\mathrm{S}$ laws with the variations in the strain rate, respectively. 


\section{RESULTS AND CONCLUSIONS}

EPDM film is an advanced insulation material; layers of EPDM films can be entwined to form a laminated composite which is very important for the structural integrity of the Solid Rocket Motor. To study the anisotropic mechanical behavior of EPDM film, specimens were cut along different directions, and rotated by 7 different angles from the orientation of the fibers, and these specimens were tested with uniaxial elongations. On each occasion of the rotating angles, four loading speeds were applied to the specimens. In this way the rate-dependency of the material was also investigated. Using the classic Tsai-Hill strength criterion, we substituted for the constant strengths with the ratedependent function forms, which fitted to an exponential form and used with the strength obtained from the experiments, then a rate-dependent Tsai-Hill strength criterion can be built. The study also described the method of introducing a rate-dependency factor into the criterion to make it workable for EPDM films. The results from comparing the predicted values with the experimental values showed a good agreement, which verified the validity of the rate-dependent orthotropic criterion. The study may serve as an essential fundamental study for investigating the laminated EPDM in the future, and provide essential parameters for numerical analyses of the SRM.

\section{ACKNOWLEDGEMENTS}

This work was supported by Natural Science Foundation of Jiangsu Province (No. BK20140772), the Fundamental Research Funds for the Central Universities (No. 30915011301, No. 30915118805). We thank LetPub (www.letpub.com) for its linguistic assistance during the preparation of this manuscript.

\section{REFERENCES}

[1] Khan MB. An investigation of the ablation behavior of advanced ultrahigh-temperature EPDM/epoxy insulation composites. Polymer-Plastics Technol Eng 1996; 35(1): 187-206.

[2] Azzi V, Tsai S. Anisotropic strength of composites. Exp Mech 1965; 5(9): 283-8.

[3] Hill R. The mathematical theory of plasticity, Oxford University Press, London, 1950: 317.

[4] Cheng M, Chen W. Experimental investigation of the stress-stretch behavior of EPDM rubber with loading rate effects. Int J Solids Struct 2003; 40(18): 4749-68.

[5] Song B, Chen W. One-dimensional dynamic compressive behavior of EPDM rubber. J Eng Mat Technol 2003: 125(3): 294-301.

[6] Le lay F. Study on the lifetime of EPDM seals in nuclear-powered vessels. Radiat Phys Chem 2013; 84(0): 210-7.

[7] Usuki A,Tukigase A, Kato M. Preparation and properties of EPDM-clay hybrids. Polymer 2002; 43(8): 2185-9.

\section{CONFLICT OF INTEREST}

The authors confirm that this article content has no conflict of interest. 